\title{
Camptodactyly-Arthropathy-Coxa Vara-Pericarditis Syndrome Resembling Juvenile Idiopathic Arthritis: A Single-Center Experience from Southern Turkey
}

\author{
Rabia Miray Kisla Ekinci ${ }^{a}$ Sibel Balci ${ }^{a}$ Haldun Dogan ${ }^{b}$ Serdar Ceylaner ${ }^{b}$ \\ Celal Varan ${ }^{c}$ Sevcan Erdem ${ }^{c}$ Fatma Coban ${ }^{d}$ Atil Bisgin $^{d}$ \\ aDepartment of Pediatric Rheumatology, Cukurova University Faculty of Medicine, Adana, Turkey; \\ bINTERGEN Genetic Diagnosis and Research Center, Ankara, Turkey; ' Department of Pediatric Cardiology, \\ Cukurova University Faculty of Medicine, Adana, Turkey; ${ }^{\text {dDepartment }}$ of Medical Genetics, AGENTEM, \\ Cukurova University Faculty of Medicine, Adana, Turkey
}

\section{Established Facts}

- Camptodactyly-arthropathy-coxa vara-pericarditis (CACP) syndrome is a monogenic disorder, caused by biallelic pathogenic mutations in the Proteoglycan 4 (PRG4) gene.

- The causative PRG4 gene encodes lubricin, which is highly expressed in synovial tissue and involved in cell growth and lubrication of joint and tendon surfaces.

\section{Novel Insights}

- In this study, we report 3 patients with CACP syndrome, of which all had camptodactyly since the first years of their lives, and 2 had a prior diagnosis of juvenile idiopathic arthritis.

- Hip changes were present in 2 patients, and pericarditis was not found in any patients.

\section{Keywords}

Arthropathy · Camptodactyly · Coxa vara · Pericarditis .

Proteoglycan 4

\section{Abstract}

Camptodactyly-arthropathy-coxa vara-pericarditis (CACP) syndrome, caused by biallelic pathogenic mutations in the PRG4 gene, is characterized by early-onset camptodactyly, noninflammatory arthropathy, coxa vara deformity, and rarely, pericardial effusion. Herein, we report 3 patients with CACP syndrome from 2 unrelated families. All patients are female, born to consanguineous parents, and had camptodactyly since the first years of their lives. Two patients had a prior diagnosis of juvenile idiopathic arthritis. Hip changes were present in 2 patients, and 2 of 3 patients had undergone surgery for camptodactyly. Routine echocardiographic evaluations were normal during the 2-year follow-up. This karger@karger.com

(C) 2021 S. Karger AG, Basel

www.karger.com/msy

Karger ${ }^{\prime}=$
Rabia Miray Kisla Ekinci

Department of Pediatric Rheumatology, Cukurova University Faculty of Medicine Saricam, Adana

01260 (Turkey)

mir_kisla@hotmail.com 
paper represents the third study including CACP patients from Turkey. Clinically, all 3 patients resembled juvenile idiopathic arthritis cases and received unnecessary medication. There is also an ongoing need for improving awareness of CACP and an effective treatment focusing on the lubrication of the joint space in CACP patients.

(C) 2021 S. Karger AG, Basel

\section{Introduction}

Camptodactyly-arthropathy-coxa vara-pericarditis (CACP) syndrome was first described in 1965 and only few patients had been reported until the genetic background of the disease was described [Jacobs and Downey, 1974; Bulutlar et al., 1986]. The main clinical characteristics of CACP syndrome are camptodactyly presenting in early childhood, noninflammatory arthropathy, a debilitating coxa vara deformity, and occasionally, pericardial effusion. Since the manifestations became more prominent in time, patients had been usually misdiagnosed as juvenile idiopathic arthritis and received immunosuppressive medication and physiotherapy prior to CACP diagnosis [Taşar et al., 2014]. The causative Proteoglycan 4 (PRG4) gene, encoding lubricin which is highly expressed in synovial tissue and involved in cell growth and lubrication of joint and tendon surfaces, was identified in 2000 [Ikegawa et al., 2000; Ciullini Mannurita et al., 2014]. Through its binding to the toll-like receptor, lubricin was also suggested to have an anti-inflammatory role in osteoarthritis and rheumatoid arthritis [Alquraini et al., 2015] On the contrary, it was also postulated that lubricin may be responsible for leukocyte recruitment to the inflamed joint by carrying inflammatory oligosaccharide epitopes [Estrella et al., 2010; AlSharif et al., 2015].

Besides the doubt about pathogenetic mechanisms of mutant lubricin, numerous patients with homozygote PRG4 mutations have been linked to CACP syndrome in case series so far. The majority of the patients demonstrate the camptodactyly-arthropathy-coxa vara triad; however, pericarditis is occasionally present at the time of diagnosis [Marcelino et al., 1999; Albuhairan and AlMayouf, 2013; Madhusudan et al., 2016]. In a recent paper, 1 of the 4 CACP patients was reported to have bilateral congenital cataracts. The authors postulated that lubricin deficiency could be related to cataract in that case. Furthermore, another study approved that lubricin is also expressed in ocular epithelial cells [Akawi et al., 2012; Schmidt et al., 2013]. Hence, reporting new pa- tients may help explain unknown manifestations of CACP syndrome and clarify a possible phenotypic variability. Therefore, herein we report a single-center experience of 3 patients with CACP syndrome from 2 families; 1 patient had sensorineural hearing loss (SNHL) in addition to camptodactyly-arthropathy-coxa vara triad.

\section{Case Presentations}

\section{Patient 1}

An 8-year-old female, born to consanguineous parents, was admitted to our department with swelling and intermittent pain in both knees and ankles for the last 4 months. Additionally, she had been suffering from finger contractures since infancy. Initial physical examination revealed significant camptodactyly of the hands, prominent swelling of both knees, warmth and a decreased range of motion (ROM) in elbows and ankles, and markedly limited hip ROM (Fig. 1a, b). Otherwise, the systemic examination including the cardiovascular system was normal. Laboratory results showed normal acute phase reactants and the absence of autoantibodies. An anteroposterior pelvic radiograph revealed bilateral subchondral sclerosis and irregularity of acetabular borders (Fig. 2a). Plain hand radiographs revealed a significant loss in joint spaces of proximal interphalangeal (PIP) joints, flexion deformities in PIP joints of the 1st, 4th, and 5th fingers of the left hand and 1st and 5th fingers of the right hand (Fig. 2b). Magnetic resonance imaging (MRI) of the knees showed synovial thickening and bilateral large joint effusion spaces, extending to the suprapatellar region (Fig. 2c). Therefore, we diagnosed the patient with polyarticular juvenile idiopathic arthritis (JIA) and initiated subcutaneous methotrexate treatment weekly (15 mg/m $\mathrm{m}^{2}$ dose) and ibuprofen (30 mg/kg per day). Furthermore, intra-articular triamcinolone acetate injection was applied to both knees. Since the patient did not respond after 6 months of this treatment, weekly etanercept, an anti-tumor necrosis alpha (anti-TNFa) agent, was added to her treatment. Six months later, etanercept was switched to another anti-TNF. Infliximab was given every 6 weeks regarding the persistence of swollen joints on previous treatment. Despite 4 doses of infliximab, concomitant with methotrexate and ibuprofen treatment, we could not achieve remission. Therefore, we considered CACP syndrome, although the patient had several inflammatory signs on her elbows and ankles. Next-generation sequencing (NGS) of the PRG4 gene showed a homozygote missense mutation, NM_005807.5(PRG4):c.538C>T; (p.Arg180Trp). Although this variant was interpreted as benign in the Clinvar database, we further identified this variant as disease-causing according to in silico tools, including SIFT, PolyPhen-2, and Provean. The patient was eventually diagnosed with CACP syndrome at the age of 10 years, and previous anti-inflammatory medication were all ceased, only ibuprofen was prescribed for symptomatic relief, and intensive physiotherapy was started. Serum 25-hydroxy-vitamin $\mathrm{D}(\mathrm{ng} / \mathrm{mL})$ level and bone mineral density were found low, and therefore we started vitamin D and calcium supplementation. Echocardiography revealed normal pericardium at diagnosis and during the 2-year follow-up. 

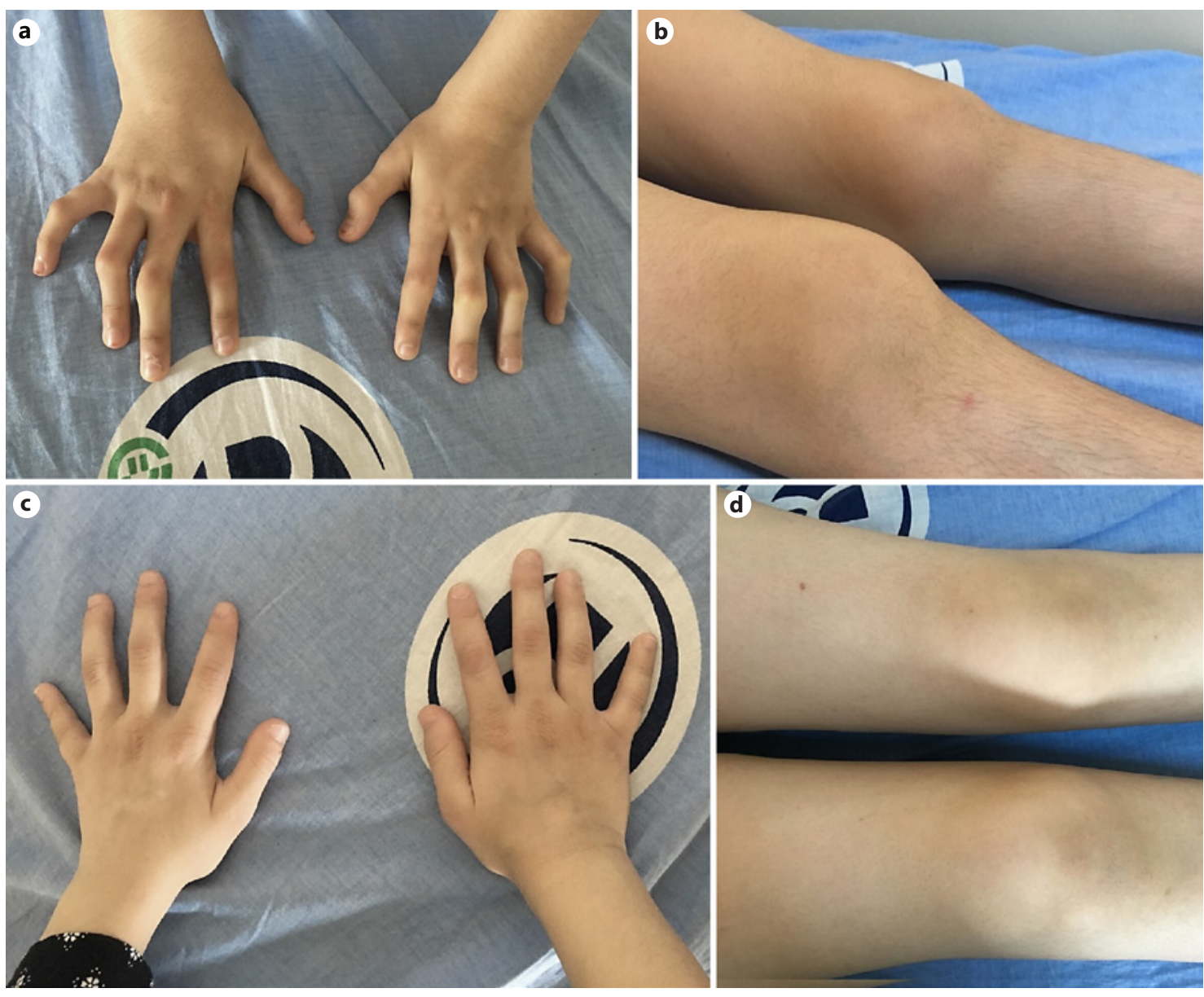

Fig. 1. Photographs of patient 1 and 2. a Prominent camptodactyly of Patient 1 . b Swelling of both knees in Patient 1. c Mild camptodactyly of the left 3rd and 4th proximal interphalangeal (PIP) joints and right 1st, 2nd, 3rd PIP joints. d Prominent swelling of both knees in Patient 2.

\section{Patient 2}

A 6-year-old female patient, from a consanguineous family, was referred to our department with bilateral knee swelling, and morning stiffness in the small joints of both hands for the past 6 months, lasting for $15 \mathrm{~min}$. Medical history revealed previous surgery, which had been performed 1 year before, for the contractures of her thumbs. The patient also had persistent unilateral SNHL, without a history of head injury or ototoxic medication, at the age of 5 years. Initial musculoskeletal examination showed warm, tender, and swollen knees, and slightly decreased ROM in the left 3rd and 4th proximal interphalangeal (PIP) joints and right 1st, 2nd, and 3rd PIP joints (Fig. 1c, d). Hip movements were markedly restricted and painful. Pelvis radiography showed coxa vara and acetabular irregularity. Acute phase reactants were normal and autoantibodies, including antinuclear antibody and rheumatoid factor were also negative in laboratory investigations. MRI showed moderate effusion in the bilateral suprapatellar bursa and hip joints and bilateral bone cysts at the acetabular surface (Fig. 2d, e). The patient was diagnosed with JIA and was treated with methotrexate and ibuprofen consequently. Since the patient did not respond to 6 months of that treatment, etanercept was also initiated weekly. The pain intermittently continued, knee swelling persisted, and the patient did suffer from new elbow swelling afterward; thus, we continued the anti-inflammatory medication. Due to the lack of favorable response to previous treatment, we considered CACP and performed NGS of the PRG4 gene, which revealed a homozygote mutation, NM_005807.6(PRG4):c.1194delC (p.Thr399ProfsTer513), classified as pathogenic in the ClinVar database, at the age of 13 years. As this is a frameshift variant, it was classified as pathogenic by the American College of Medical Genetics and Genomics (ACMG) criteria. Null variant (frameshift) in the PRG4 gene, for which loss of function (LOF) is a known mechanism of disease (the gene has 17 pathogenic LOF variants and the LOF $Z$ Score $=2.8$ is greater than 0.7 ), associated with CACP syndrome.

Following, we ceased all medication except for ibuprofen, which may bring symptomatic pain relief, and intensive physiotherapy was initiated. We also started vitamin $\mathrm{D}$ supplementation due to the low serum 25-hydroxy-vitamin D (ng/mL) level, although bone mineral density was normal. Echocardiographic investigation was normal at diagnosis and during the 2-year followup. In this period, we also investigated the patient for known 

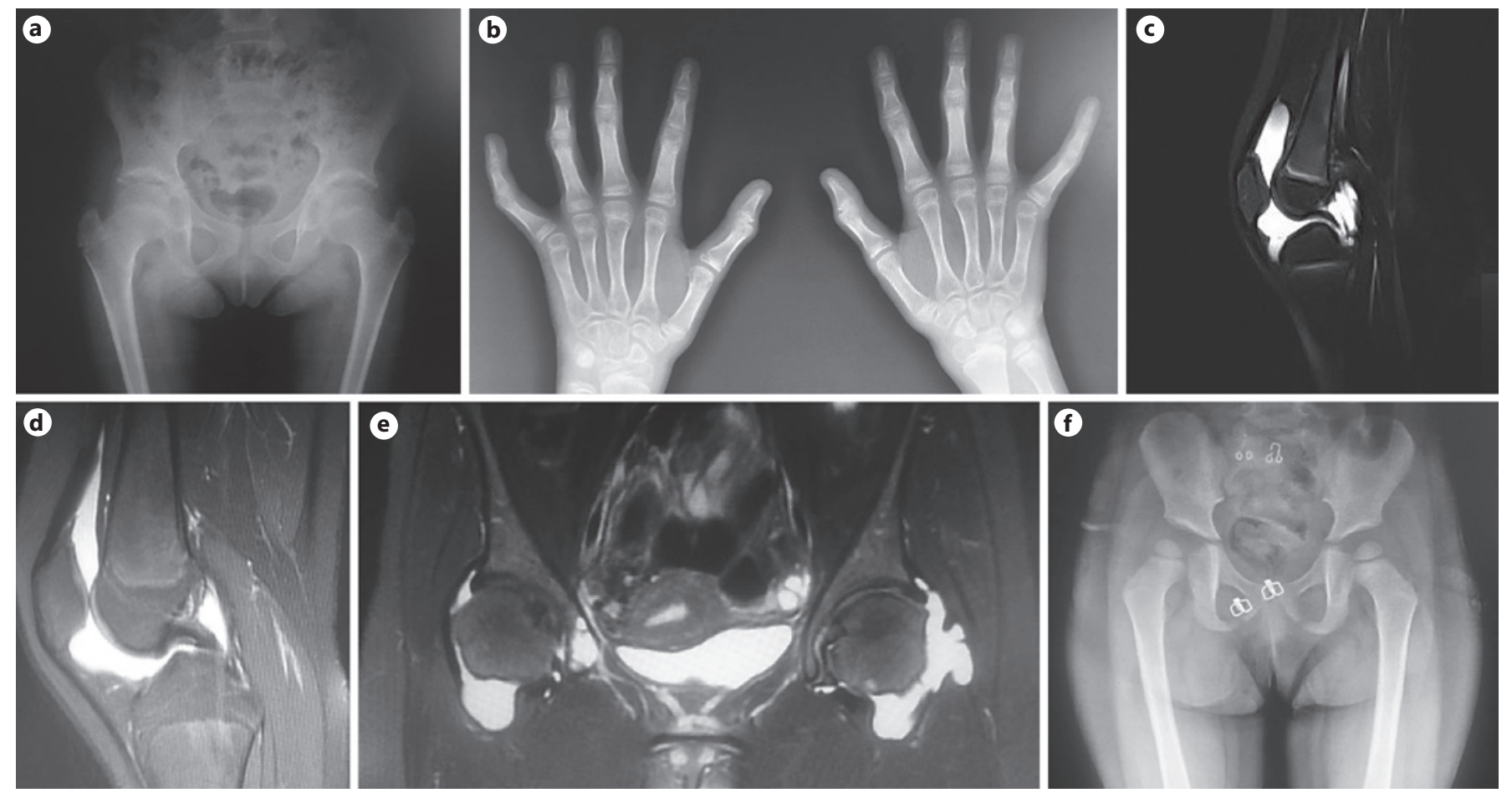

Fig. 2. Radiological evaluation of patients with camptodactyly-arthropathy-coxa vara-pericarditis syndrome. a Anteroposterior (AP) pelvis radiograph of Patient 1 showing bilateral subchondral sclerosis, and irregularity of acetabular borders. b Plain radiographs of hands revealing significant loss in joint spaces of proximal interphalangeal (PIP) joints, flexion deformities in PIP joints of the 1st, 4th, and 5th fingers of the left hand, and 1st and 5th fingers of the right hand of Patient 1. c MRI of Patient 1 showing synovial thickening and bilateral large joint effusion spaces, extending to the suprapatellar region of the left knee. $\mathbf{d}$ MRI images of Patient 2 showing moderate effusion in bilateral suprapatellar bursa. e MRI of Patient 2 revealing prominent effusion in bilateral hip joints and bone cysts at the acetabular surface. $\mathbf{f}$ Normal AP pelvis graph of Patient 3. causes of SNHL, including multiple sclerosis, vasculitis, inner ear malformation, and central nervous system malignities, but no relevant causes were identified.

\section{Patient 3}

A 3-year-old female patient, the sibling of Patient 2, had undergone orthopedic surgery for flexion contracture on the 1st, 3rd, and 4 th fingers of both hands at the age of 1 year. She had no other skeletal complaints. Her physical examination was normal except for mild knee swelling without being warm, tender, or showing restriction of ROM. Anteroposterior pelvic radiograph was normal (Fig. 2f). Echocardiogram was normal during the followup. We simultaneously performed NGS for the PRG4 gene and identified the same NM_005807.5(PRG4):c.1194del (p.Thr399fs) mutation, of which the homozygosity was again confirmed by homozygosity mapping. Since the patient did not suffer from pain, we did not initiate any anti-inflammatory medication. We gave vitamin D supplementation since the serum 25-hydroxy-vitamin $\mathrm{D}(\mathrm{ng} / \mathrm{mL})$ level was at the lower border of normal. The clinical features of the patients described in this paper are summarized in Table 1.

\section{Materials and Methods}

PRG4 gene sequencing analysis was performed using the MiSeq NGS platform (Illumina, San Diego, CA, USA). Genomic DNA of all the family members was extracted according to the manufacturer's standard procedure using the QIAamp DNA Blood Midi Kit (Qiagen, Hilden, Germany). The DNA samples were quantified with a NanoDrop 1000 (Thermo Fisher Scientific Inc.) spectrophotometer and used at a concentration of $50 \mathrm{ng} / \mu \mathrm{L}$. All coding exons and their flanking splice site junctions were amplified using PCR primers, designed with PRIMER ${ }^{\odot}$ - Primer Designer v.2.0 (Scientific \& Educational Software program) software. PCRs were validated by using agarose gel electrophoresis. PCRs for each individual were mixed to obtain PCR pools, purified and quantified. Purifications were done by using NucleoFast ${ }^{\circledR} 96$ PCR kiti (MACHEREY-NAGEL GmbH) and quantification of purified PCRs were done by using NanoDrop 1000. Quantified PCR pools then standardized to $0.2 \mathrm{ng} / \mathrm{ul}$. The libraries were prepared with the NexteraXT kit (Illumina Inc.), according to the manufacturer's instructions. NGS was carried out on MiSeq (Illumina Inc.). Sequences were aligned to the hg19 genome within MiSeq Reporter software (Illumina Inc.). Visualization of the data was performed with IGV 2.3 (Broad Institute) software. 
Serum was obtained from the blood samples of the patients by centrifugation at 2,500 rpm for $5 \mathrm{~min}$. Lubricin protein analysis from serum samples was performed by using the enzyme-linked immunosorbent assay (ELISA) method. Proteoglycan 4 (PRG4)/ Lubricin ELISA kit (USCN, Wuhan) was used for protein analysis. ELISA plates were analyzed with BIOTEK 800 TS Absorbance Reader.

\section{Results}

Serum lubricin levels of patient 1,2 , and 3 were 0.553 , 0.107 , and $0.457 \mathrm{U} / \mathrm{mL}$, respectively. The mean serum lubricin levels of a control group, including 28 healthy children, were also analyzed as $0.99 \pm 0.514$. The results of all patients were lower than healthy controls.

\section{Discussion}

Lubricin is a mucin-rich glycoprotein, secreted by synovial fibroblasts and chondrocytes, thus, has homeostatic roles on multiple tissues including articular cartilage, meniscus, and tendons in humans. Besides its estimated overall lubricating role in joints, it is still not clear whether it acts as an inflammatory factor or prevents inflammatory damage [Estrella et al., 2010; Al-Sharif et al., 2015; Alquraini et al., 2015]. Misdiagnosing CACP patients as JIA has been common so far, similar to the treatment of a substantial proportion of CACP patients with antirheumatic drugs and even biological agents without any significant response. In a large series of 22 CACP patients, the mean age at diagnosis was 15.6 years, and all patients had camptodactyly and coxa vara with radiological changes, whereas pericarditis was present in only 2 children aged 5 years. Arthropathy of CACP typically involves large joints, including wrist, elbow, hips, knee, and ankles. The same study revealed a previous medication history of antirheumatic drugs in 10 CACP patients, and 2 of them also received etanercept, an anti-tumor necrosis-alpha agent [Albuhairan and Al-Mayouf, 2013]. Another case series from Saudi Arabia, included 7 CACP patients, diagnosed between 18 months and 8 years of age. None of them showed pericarditis, while camptodactyly was the initial sign, usually presented in the first months of life [Alazami et al., 2006]. Consanguinity plays a major role in the development of inherited autosomal recessive diseases, including CACP; in this regard, a recent study identified 6 affected individuals from a large consanguineous Pakistani family. All patients bear a novel homozygous deletion in the PRG4 gene resulting in a frameshift, and none of them developed pericarditis in their follow-up [Basit et al., 2011]. On the contrary, 2 patients aged 9 and 13 years, with another homozygous deletion in the PRG4 gene, presented with pericarditis at diagnosis [Patil et al., 2016].

Similar to the literature, our patients had been diagnosed with JIA, and 2 of 3 patients were taking antirheumatic medication including intra-articular steroid, methotrexate, and etanercept before the actual CACP diagnosis. The initial clinical sign was camptodactyly in all patients, while arthropathy of knees and coxa vara were progressive and nonresponding to current treatment. We also supported the genetic diagnosis of the patients because of low serum lubricin levels, revealed by ELISA.

This paper represents the third study including CACP patients from Turkey. Bulutlar et al. [1986] were the first to report this disorder in 4 sisters. Yilmaz et al. [2018] followed, describing the clinical findings of CACP syndrome in 35 patients from 11 unrelated families, 10 of which were from the southeast region of Turkey. In the latter study, nearly half of the patients were earlier misdiagnosed as JIA. Age at diagnosis was between 3.5 and 53 years, while age at onset was 1-24 months [Yilmaz et al., 2018]. Therefore, we think that the physicians still underestimate the possibility of CACP diagnosis, a pseudo-JIA disease in the differential diagnosis of childhood arthropathies. The other major concern is that without the accurate diagnosis of CACP in clinical practice, we cannot adequately treat the patients with an available curative drug, such as intra-articular recombinant lubricin for CACP.

Although we report 3 cases with CACP, it is important to show that 2 of them were diagnosed as JIA before the exact diagnosis. We did not identify pericarditis in routine echocardiographic follow-up, and none of the patients exhibited cardiac symptoms such as sudden chest pain or palpitation. It was interesting to show SNHL in one of our patients with no other identifiable cause; however, we cannot be sure whether SNHL was due to CACP or not because there is no information related to the expression of lubricin in the human inner ear. Therefore this association could be clarified by further work on both functional studies and reports of a large number of CACP patients.

Al-Mayouf et al. [2017] recently investigated the effectiveness of radiosynovectomy on 6 children with CACP. Although this procedure was defined as safe and welltolerated, this study did not show any benefits on arthropathy of CACP. Therefore, we also wish to emphasize the need for an effective treatment protocol in CACP with the aim of joint space lubrication. 
In conclusion, herein, we described 3 CACP patients, presenting with chronic arthritis during early childhood. With this report, we wish to highlight the need for early recognition of CACP during differential diagnosis of childhood arthropathies, especially in the presence of camptodactyly.

\section{Acknowledgements}

We sincerely thank our mentor, Prof. Mustafa Yilmaz, for his great support on our pediatric rheumatology education. We are also grateful for his contribution to our professional life. He passed away last year, but he will be in our memories for the rest of our lives.

\section{Statement of Ethics}

The patients and their parents have given their written informed consent to publish their case, including publication of images. The study was conducted in accordance with the ethical standards released in the 1964 Declaration of Helsinki and its later amendments.

\section{Conflict of Interest Statement}

The authors have no conflicts of interest to declare.

\section{Funding Sources}

No funding was received.

\section{Author Contributions}

Dr. Kisla Ekinci and Dr. Bisgin conceptualized and designed the study, drafted the initial manuscript, and reviewed and revised the manuscript. Dr. Balci, Dr. Erdem, Dr Coban, and Dr. Varan collected data, carried out the initial analyses, and critically reviewed and revised the manuscript. Dr. Dogan and Dr. Ceylaner performed the genetic analysis. All authors approved the final manuscript as submitted and agree to be accountable for all aspects of the work. All co-authors take full responsibility for the integrity of the study.

\section{References}

Akawi NA, Ali BR, Al-Gazali L. A novel mutation in PRG4 gene underlying camptodactyly-arthropathy-coxa vara-pericarditis syndrome with the possible expansion of the phenotype to include congenital cataract. Birth Defects Res Part A Clin Mol Teratol. 2012;94(7):5536.

Al-Mayouf SM, Almutairi N, Alismail K. The Efficacy of Yttrium-90 Radiosynovectomy in Patients with Camptodactyly-ArthropathyCoxa Vara-Pericarditis Syndrome. Mol Imaging Radionucl Ther. 2017;26(1):33-7.

Al-Sharif A, Jamal M, Zhang LX, Larson K, Schmidt TA, Jay GD, et al. Lubricin/Proteoglycan 4 Binding to CD44 Receptor: A Mechanism of the Suppression of Proinflammatory Cytokine-Induced Synoviocyte Proliferation by Lubricin. Arthritis Rheumatol. 2015;67(6): 1503-13.

Alazami AM, Al-Mayouf SM, Wyngaard CA, Meyer B. Novel PRG4 mutations underlie CACP in Saudi families. Hum Mutat. 2006; 27(2):213.

Albuhairan I, Al-Mayouf SM. Camptodactyly-arthropathy-coxavara-pericarditis syndrome in Saudi Arabia: clinical and molecular genetic findings in 22 patients. Semin Arthritis Rheum. 2013;43:292-6.

Alquraini A, Garguilo S, D'Souza G, Zhang LX, Schmidt TA, Jay GD, et al. The interaction of lubricin/proteoglycan 4 (PRG4) with toll-like receptors 2 and 4: an anti-inflammatory role of PRG4 in synovial fluid. Arthritis Res Ther. 2015; 17:353.

Basit S, Iqbal Z, Umicevic-Mirkov M, Kamran UlHassan Naqvi S, Coenen M, Ansar M, et al. A novel deletion mutation in proteoglycan-4 underlies camptodactyly-arthropathy-coxavara-pericarditis syndrome in a consanguineous pakistani family. Arch Med Res. 2011; 42(2):110-4.

Bulutlar G, Yazici H, Ozdoğan H, Schreuder I. A familial syndrome of pericarditis, arthritis, camptodactyly, and coxa vara. Arthritis Rheum. 1986;29(3):436-8.

Ciullini Mannurita S, Vignoli M, Bianchi L, Kondi A, Gerloni V, Breda L, et al. CACP syndrome: identification of five novel mutations and of the first case of UPD in the largest European cohort. Eur J Hum Genet. 2014;22(2): 197-201.

Estrella RP, Whitelock JM, Packer NH, Karlsson NG. The glycosylation of human synovial lubricin: implications for its role in inflammation. Biochem J. 2010;429(2):359-67.

Ikegawa S, Sano M, Koshizuka Y, Nakamura Y. Isolation, characterization and mapping of the mouse and human PRG4 (proteoglycan 4) genes. Cytogenet Cell Genet. 2000;90(3-4): 291-7.

Jacobs JC, Downey JA. The child with disabling illness. In: Downey JA, Low NLeditors. Juvenile Rheumatoid Arthritis.Philadelphia: Saunders; 1974. p. 5-24.
Madhusudan S, Gupta A, Prakash M, Matta D, Suri D, Singh S. Camptodactyly-arthropathycoxa vara-pericarditis (CACP) syndrome: a mimicker of juvenile idiopathic arthritis. Scand J Rheumatol. 2016;45(1):77-8.

Marcelino J, Carpten JD, Suwairi WM, Gutierrez OM, Schwartz S, Robbins C, et al. CACP, encoding a secreted proteoglycan, is mutated in camptodactyly-arthropathy-coxa vara-pericarditis syndrome. Nat Genet. 1999;23(3): 319-22.

Patil DV, Phadke MS, Pahwa JS, Dalal AB. Brothers with constrictive pericarditis - A novel mutation in a rare disease. Indian Heart J. 2016;68(Suppl 2):S284-7.

Schmidt TA, Sullivan DA, Knop E, Richards SM, Knop N, Liu S, et al. Transcription, translation, and function of lubricin, a boundary lubricant, at the ocular surface. JAMA Ophthalmol. 2013;131(6):766-76.

Taşar M, Eyileten Z, Kasımzade F, Uçar T, Kendirli T, Uysalel A. Camptodactyly-arthropathy-coxa vara-pericarditis (CACP) syndrome. Turk J Pediatr. 2014;56(6):6846.

Yilmaz S, Uludağ Alkaya D, Kasapçopur Ö, Barut K, Akdemir ES, Celen C, et al. Genotype-phenotype investigation of 35 patients from 11 unrelated families with camptodactyly-arthropathy-coxa vara-pericarditis (CACP) syndrome. Mol Genet Genomic Med. 2018; 6(2):230-48. 